\title{
Synthesis of a 7-(aminomethyl)indole and related bis-indole derivatives
}

\author{
Hakan Kandemir, ${ }^{\text {a,b }}$ Ibrahim F. Sengul,,${ }^{\text {acc }}$ Naresh Kumar, ${ }^{\text {a }}$ and David StC. Black*a \\ ${ }^{a}$ School of Chemistry, University of New South Wales, Sydney, NSW 2052, Australia \\ ${ }^{b}$ Department of Chemistry, Faculty of Art and Science, Namık Kemal University, Tekirdag, \\ Turkey \\ ${ }^{c}$ Department of Chemistry, Faculty of Science, Gebze Technical University, Kocaeli, Turkey \\ E-mail: d.black@unsw.edu.au
}

DOI: $\underline{\text { http://dx.doi.org/10.3998/ark.5550190.p009.587 }}$

\begin{abstract}
A 7-(aminomethyl)indole is a useful precursor to form a new range of unsymmetrical and symmetrical 3-substituted amide-, imine-, and amine-linked 7,7'-bis-indoles. The reduction of an imine linked bis-indole leads to formation of the corresponding amine-linked bis-indole.
\end{abstract}

Keywords: Indoles, bis-indoles. 7-aminomethylindoles, 7-formylindoles, 7-cyanoindoles

\section{Introduction}

The aminomethyl moiety is found in many indole alkaloids which possess biological activity. ${ }^{1}$ For example, brassinin 1 and cyclobrassinin 2, are representative members of the phytoalexin family that have been isolated from cabbage and exhibit antitumor activity. ${ }^{2}$ During the last two decades, approximately thirty phytoalexins have been isolated from cruciferous plants. The key intermediate for the synthesis of the phytoalexins is 3 -aminomethylindole $3 .^{3}$

More complex indoles such as bis-indoles are also very important biologically active scaffolds as they are found in many pharmacologically active alkaloids. ${ }^{4}$ Moreover, aminomethylindole based bis-indoles have been isolated from natural sources and demonstrate potential as biologically active compounds and useful synthetic targets. For example, bis-indole 4 has been isolated from the roots of Antirhea lucida, and synthesized from tryptamine derivatives through acid catalysed nucleophilic substitution of 1-hydroxytryptamine. ${ }^{5}$

Given the various potential applications of bis-indoles, it is important to develop new classes of natural and unnatural bis-indole derivatives. This development can be greatly facilitated by the use of activated indoles which are capable of undergoing reaction at the C7 position. 


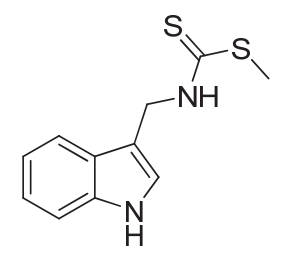

1

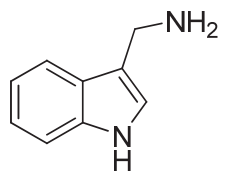

3
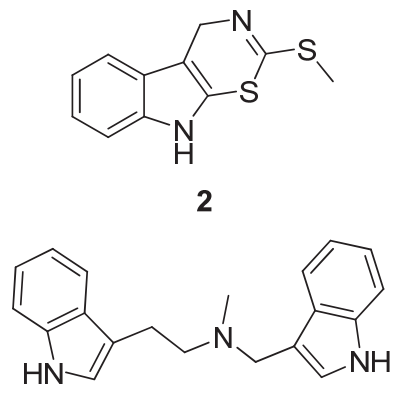

4

Figure 1. Examples of biologically active aminomethylindoles and derived compounds.

\section{Results and Discussion}

Reduction of the 7-cyanoindole ${ }^{6} \mathbf{5}$ was carried out using lithium aluminium hydride in tetrahydrofuran and gave exclusively the 7-aminomethylindole 6 in $73 \%$ yield (Scheme 1).

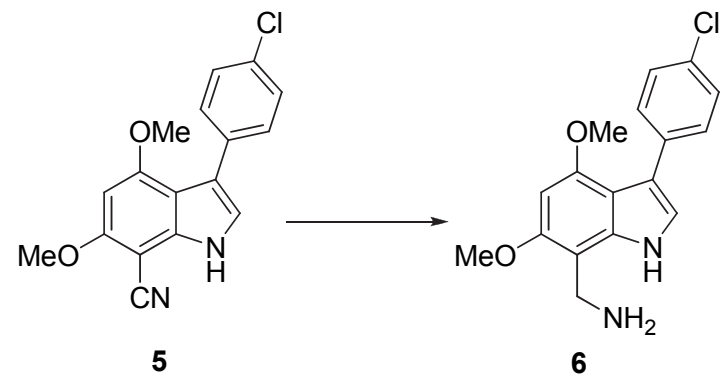

Scheme 1. Reagent and conditions: $\mathrm{LiAlH}_{4}, \mathrm{THF}$, rt.

The infrared spectrum of the amine 6 showed the characteristic absorptions of the primary amino group at 3368 and $3360 \mathrm{~cm}^{-1}$. The formation of the amine was also deduced from the alkyl protons being present in the ${ }^{1} \mathrm{H}$ NMR spectrum at $4.15 \mathrm{ppm}$ and a $\mathrm{CH}_{2}$ signal appearing in the DEPT135 experiment. An $\mathrm{m} / \mathrm{z}$ of 316.0973 was also obtained via high resolution mass spectrometry that was consistent with the target structure $\mathbf{6}$.

For the construction of 7,7'-linked-bis-indoles two basic strategies have been used. The first involves the formation of symmetrical and unsymmetrical bis-indoles by joining the 7aminomethyindole scaffold to mono- and bi-functional acyl chloride linkers such as 7trichloroacetylindole, oxalyl chloride and adipoyl chloride. The amide functionality is one of the most prevalent structural moieties present in polymers, natural products and pharmaceuticals. ${ }^{7}$ Accordingly, the first target 7,7'-bis-indole carboxamide 8 was prepared in $83 \%$ yield by the 
reaction of equimolar amounts of 7-aminomethyindole 6 and 7-trichloroacetylindole $7^{8}$ in hot acetonitrile (Scheme 2).

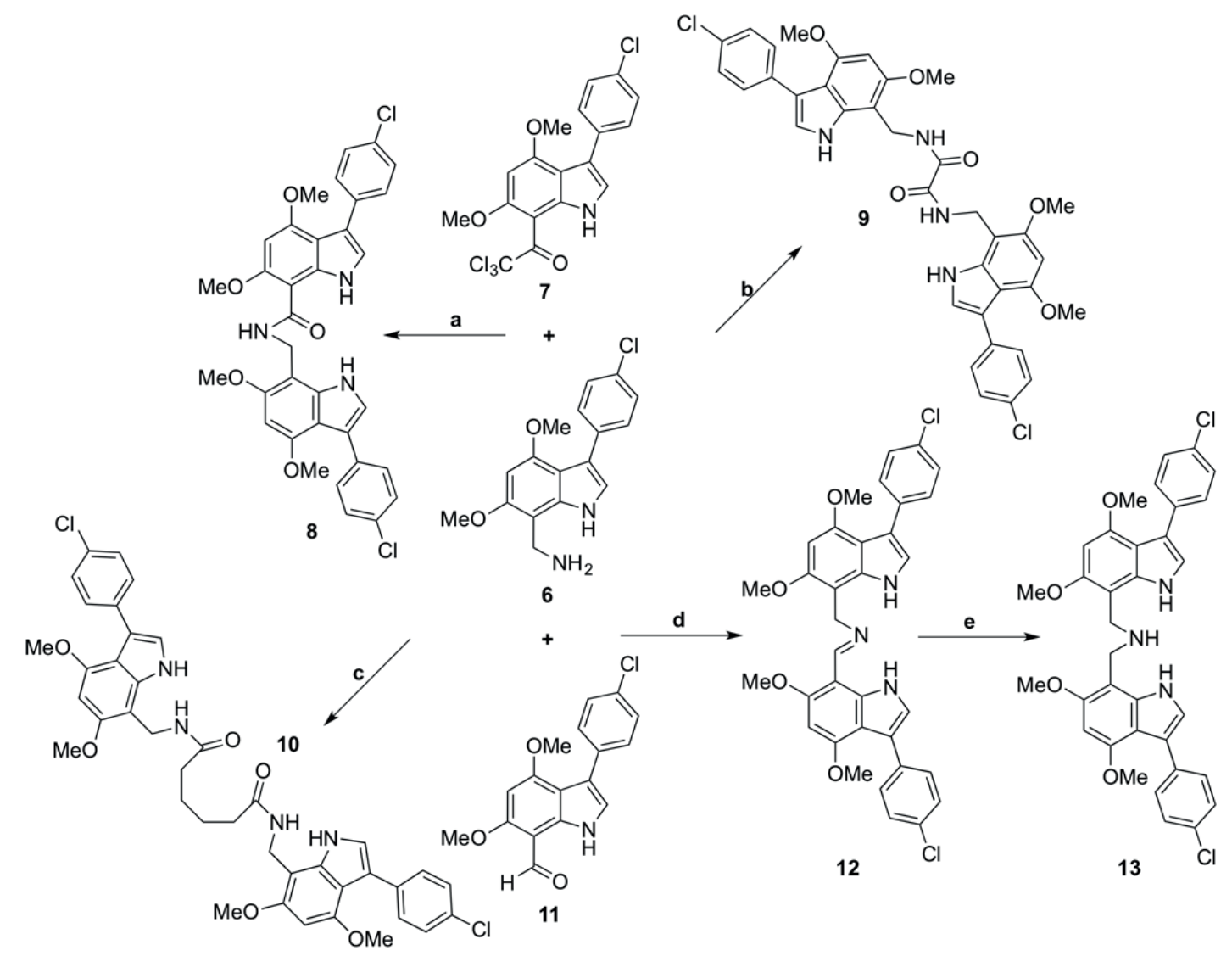

Scheme 2. Reagent and conditions: (a) $\mathrm{Et}_{3} \mathrm{~N}, \mathrm{CH}_{3} \mathrm{CN}$, reflux, overnight; (b) oxalyl chloride, $\mathrm{Et}_{3} \mathrm{~N}$, DCM, r.t., 1 h; (c) adipoyl chloride, $\mathrm{Et}_{3} \mathrm{~N}$, DCM, r.t., 1 h; (d) absolute ethanol, HCl, reflux; (e) $\mathrm{NaBH}_{4}, \mathrm{THF}$, absolute ethanol, reflux.

The construction of amide linked bis-indoles $\mathbf{9}$ and $\mathbf{1 0}$ was achieved by the treatment of the indole 6 with 0.5 equivalents of oxalyl chloride and adipoyl chloride respectively in the presence of triethylamine in dichloromethane. Both of the reactions were complete in an hour and the bisindoles 9 and 10 were afforded in $81 \%$ and 86\% yield respectively. In the ${ }^{1} \mathrm{H}$ NMR spectrum of compound 10, for example, the amide $\mathrm{NH}$ appeared as a triplet at $8.27 \mathrm{ppm}$, while the indole $\mathrm{NH}$ resonance was observed at $10.91 \mathrm{ppm}$. The high chemical shift is indicative of strong intramolecular hydrogen bonding. Compound $\mathbf{1 0}$ also showed good solubility in deuterated dimethyl sulfoxide, allowing observation of a carbonyl resonance at $173 \mathrm{ppm}$ in the ${ }^{13} \mathrm{C}$ NMR spectrum. Moreover, the $\mathrm{CH}_{2}$ group resonance was determined both with ${ }^{13} \mathrm{C}$ DEPT135 NMR experiments.

The second strategy was to combine two different indole units which have different functionality, under suitable reaction conditions to form unsymmetrical bis-indoles. 
Consequently, the condensation of 7-aminomethylindole $\mathbf{6}$ with indole-7-carbaldehyde ${ }^{9} \mathbf{1 1}$ in ethanol gave the imine bis-indole 12 in $75 \%$ yield (Scheme 2 ). The ${ }^{1} \mathrm{H}$ NMR spectrum showed the imine proton at $8.91 \mathrm{ppm}$ and the indole $\mathrm{NH}$ resonances at $11.38 \mathrm{ppm}$ and $11.49 \mathrm{ppm}$, indicating the presence of strong hydrogen bonding within the system. The protons of the four methoxy groups appeared at 3.82, 3.89, 3.90, $3.94 \mathrm{ppm}$ and the $\mathrm{H} 5$ and $\mathrm{H} 5^{\prime}$ protons at $6.46 \mathrm{ppm}$ and $6.49 \mathrm{ppm}$ confirming the unsymmetrical nature of the structure. A high resolution mass spectrum further confirmed the structure, showing a molecular ion at $614.1599(\mathrm{M}+\mathrm{Na})^{+}$. In general, secondary amines have also been prepared from the imines via catalytic hydrogenation and reduction with sodium and alcohol. ${ }^{10}$

Following this, the desired amine $\mathbf{1 3}$ was prepared by heating the imine $\mathbf{1 2}$ with sodium borohydride at reflux in a mixture of ethanol and tetrahydrofuran (Scheme 2). The ${ }^{1} \mathrm{H}$ NMR spectrum of the compound $\mathbf{1 3}$ indicated an indole amine, as expected, in combination with the disappearance of the imine bond resonance and the presence of $\mathrm{CH}_{2}$ resonance at $4.01 \mathrm{ppm}$, which clearly indicated that the reaction occurred across the double bond as anticipated. Also, ESI mass spectral analysis showed a molecular ion at $616.1768(\mathrm{M}+\mathrm{H})^{+}$.

\section{Conclusions}

In summary, 7-aminomethylindole 6 was synthesised by reduction of the corresponding 7cyanoindole 5, and proved to be a useful precursor for a range of 7,7'-bis-indoles. The amide linked bis-indoles were obtained upon reaction of 7-aminomethylindole 6 with acyl chlorides, while an imine linked compound $\mathbf{1 2}$ was produced upon reaction with 7-formylindole 11. The corresponding amine linked bis-indole $\mathbf{1 3}$ was obtained through reduction of the imine linkage with sodium borohydride. It is noteworthy that the bis-indoles all contain a nucleophilic unsubstituted $\mathrm{C} 2$ position that provides scope for further functionalisation. This rather limited study provides the basis for a simple and general route to a wide range of potentially interesting bis-indole compounds.

\section{Experimental Section}

General. All reagents and solvents were obtained from commercial sources and appropriately purified, if necessary. Melting points were measured using a Mel-Temp melting point apparatus. Microanalyses were performed on a Carlo Erba Elemental Analyses EA 1108 at the Campbell Microanalytical Laboratory, University of Otago, New Zealand. ${ }^{1} \mathrm{H}$ and ${ }^{13} \mathrm{C}$ NMR spectra were obtained on Bruker DPX300 and Bruker DPX600 spectrometers. Mass spectra were recorded on either a Bruker FT-ICR MS (EI) or a Micromass ZQ2000 (ESI). Infrared spectra were recorded with a Thermo Nicolet 370 FTIR Spectrometer using KBr discs. Pressure column chromatography was carried out using Merck 230-400 mesh ASTM silica gel. Vacuum column 
chromatography was performed using Merck $60 \mathrm{H}$ silica gel. Gravity column chromatography was conducted using Merck 70-230 mesh ASTM silica gel, whilst preparative thin layer chromatography was carried out using Merck silica gel $773060 \mathrm{GF}^{254}$.

[3-(4-Chlorophenyl)-4,6-dimethoxy-1H-indol-7-yl]methanamine (6). A suspension of lithium aluminium hydride $(2.50 \mathrm{~g}, 65.8 \mathrm{mmol})$ in anhydrous tetrahydrofuran $(30 \mathrm{~mL})$ was cooled in an ice bath and stirred under argon while a solution of the 7-nitrile $\mathbf{5}^{6}$ (2.58 g, $\left.8.26 \mathrm{mmol}\right)$ in anhydrous tetrahydrofuran $(20 \mathrm{~mL})$ was added via a dropping funnel over $30 \mathrm{~min}$. Stirring was continued for $30 \mathrm{~min}$ with cooling, followed by stirring at room temperature for $4 \mathrm{~h}$. The mixture was recooled in ice and cautiously treated sequentially with water $(5 \mathrm{~mL}), 5 \mathrm{M} \mathrm{NaOH}(10 \mathrm{~mL})$ and water $(10 \mathrm{~mL})$. The resulting granular solid was filtered and washed with ethyl acetate. The combined organic filtrate was washed with brine, dried over anhydrous sodium sulfate and the solvent evaporated in vacuo. Recrystallisation from acetonitrile afforded the title compound 6 (1.9 g, 73\%) as brown crystals, Mp 179-181 ${ }^{\circ} \mathrm{C}$. IR (KBr): $v_{\max } 3368,3360,1623,1592,1464$, 1322, 1168, 1085, 938, $795 \mathrm{~cm}^{-1}$. UV-vis $(\mathrm{MeOH}): \lambda_{\max } 202 \mathrm{~nm}\left(\varepsilon 24,600 \mathrm{~cm}^{-1} \mathrm{M}^{-1}\right), 228$ (26,000), 281 (12,200). ${ }^{1} \mathrm{H}$ NMR (300 MHz, $\mathrm{CDCl}_{3}$ ): $\delta 3.74$ (s, 3H, OMe), 3.81 (s, 3H, OMe), 4.15 (s, 2H, $\left.\mathrm{CH}_{2}\right), 6.24$ (s, 1H, H5), 7.24, 7.46 (2d, J $8.8 \mathrm{~Hz}, 4 \mathrm{H}$, aryl H), 7.98 (s, 1H, H2), 9.92 (bs, 1H, NH). ${ }^{13} \mathrm{C}$ NMR (75 MHz, DMSO-d $): \delta 36.1\left(\mathrm{CH}_{2}\right), 55.5,57.3(\mathrm{OMe}), 89.7(\mathrm{C} 5), 123.1$ (C2), 127.8, $130.84(\operatorname{aryl} \mathrm{CH}), 108.0,110.2,116.0,129.3,130.0,135.8,138.0,152.9,153.4$ (aryl C). HRMS (+ESI): $\mathrm{C}_{17} \mathrm{H}_{17} \mathrm{Cl}_{2} \mathrm{~N}_{2} \mathrm{O}_{2}[\mathrm{M}]^{+}$requires 316.0979 , found 316.0973 .

\section{3-(4-Chlorophenyl)- $\mathrm{N}$-((3-(4-chlorophenyl)-4,6-dimethoxy-1H-indol-7-yl)-methyl)-4,6-}

dimethoxy-1H-indole-7-carboxamide (8). To a suspension of 7-aminomethylindole $6(0.50 \mathrm{~g}$, $1.57 \mathrm{mmol})$ and 7-trichloroacetylindole $7(0.68 \mathrm{~g}, 1.57 \mathrm{mmol})$ in acetonitrile $(40 \mathrm{~mL})$, triethylamine (15 drops) was added and the mixture was heated under reflux overnight. After cooling, the reaction mixture was poured into ice-water and the resulting precipitate was filtered, washed with water and recrystallised from ethanol to give the title compound $\mathbf{8}(0.82 \mathrm{~g}, 83 \%)$ as a white solid, Mp 234-236 ${ }^{\circ} \mathrm{C}$. IR (KBr): $v_{\max } 3401,3207,2942,2841,1596,1542,1452,1325$, 1218, 981, 836, 796, $600 \mathrm{~cm}^{-1}$. UV-vis (THF): $\lambda_{\max } 214 \mathrm{~nm}\left(\varepsilon 80,150 \mathrm{~cm}^{-1} \mathrm{M}^{-1}\right), 284(32,500) .{ }^{1} \mathrm{H}$ NMR (300 MHz, $\mathrm{CDCl}_{3}$ ): $\delta 3.87$ (s, 3H, OMe), 3.90 (s, 3H, OMe), 4.01 (s, 3H, OMe), 4.02 (s, $3 \mathrm{H}, \mathrm{OMe}$ ), 4.97 (d, J $6.7 \mathrm{~Hz}, 2 \mathrm{H}, \mathrm{CH}_{2}$ ), 6.26 (s, 1H, H5), 6.36 (s, 1H, H5'), 7.14 (d, J 2.31 Hz, 1H, H2), 7.19 (d, J 2.4 Hz, 1H, H2'), 7.31-7.56 (m, 8H, aryl H), 8.69 (m, 1H, NH), 10.65 (bs, $1 \mathrm{H}, \mathrm{NH}), 11.30$ (bs, $1 \mathrm{H}, \mathrm{NH}) .{ }^{13} \mathrm{C} \mathrm{NMR}\left(75 \mathrm{MHz}, \mathrm{CDCl}_{3}\right): \delta 32.9\left(\mathrm{CH}_{2}\right), 55.6,55.8,57.3,58.0$ (OMe), 87.8 (C5), 89.3 (C5'), 122.4 (C2), 122.7 (C2'), 128.0, 128.1, 131.0, 131.2, 132.6 (aryl $\mathrm{CH}), 97.8,104.0,111.0,111.4,117.2,117.6,131.6,134.8,135.3,138.9,139.9,154.5,154.6$, 157.3, 157.9 (aryl C), $169.2(\mathrm{C}=\mathrm{O})$. HRMS (+ESI): $\mathrm{C}_{34} \mathrm{H}_{29} \mathrm{Cl}_{2} \mathrm{~N}_{3} \mathrm{O}_{5}[\mathrm{M}+\mathrm{Na}]^{+}$requires 652.1382 , found 652.1373 .

Bis-((3-(4-chlorophenyl)-4,6-dimethoxyindol-7-yl)-methyl)-oxalamide (9). Oxalyl chloride $(0.05 \mathrm{~mL}, 0.60 \mathrm{mmol})$ in dry dichloromethane $(10 \mathrm{~mL})$ was added dropwise over $10 \mathrm{~min}$ to a solution of 7-aminomethylindole $6(0.31 \mathrm{~g}, 0.97 \mathrm{mmol})$ in dry dichloromethane $(18 \mathrm{~mL})$ containing triethylamine $(0.13 \mathrm{~mL}, 0.97 \mathrm{mmol})$ and the mixture was stirred at room temperature 
for $1 \mathrm{~h}$. The solvent was then evaporated and the residue was quenched with water. The resulting precipitate was filtered, dried and washed with hot methanol and $n$-hexane to yield the title compound $9(0.27 \mathrm{~g}, 81 \%)$ as a pale yellow solid. Mp 278-280 ${ }^{\circ} \mathrm{C}$. IR (KBr): $v_{\max } 3286,2935$, 1637, 1521, 1327, 1218, 1155, 1109, $798 \mathrm{~cm}^{-1}$. UV-vis (THF): $\lambda_{\max } 215 \mathrm{~nm}\left(\varepsilon 101,600 \mathrm{~cm}^{-1} \mathrm{M}^{-1}\right)$, 284 (42,450). ${ }^{1} \mathrm{H}$ NMR (300 MHz, $\left.\mathrm{CDCl}_{3}\right): \delta 3.74$ (s, 6H, OMe), 3.85 (s, 6H, OMe), $4.64(\mathrm{~d}, J$ $\left.6.9 \mathrm{~Hz}, 4 \mathrm{H}, \mathrm{CH}_{2}\right), 6.20$ (s, 2H, H5), $6.93(\mathrm{~d}, J 2.4 \mathrm{~Hz}, 2 \mathrm{H}, \mathrm{H} 2), 7.21,7.39(2 \mathrm{~d}, J 8.6 \mathrm{~Hz}, 8 \mathrm{H}$, aryl $\mathrm{H}), 7.95(\mathrm{~m}, 2 \mathrm{H}, \mathrm{NH}), 9.56(\mathrm{bs}, 2 \mathrm{H}, \mathrm{NH}) .{ }^{13} \mathrm{C} \mathrm{NMR}\left(75 \mathrm{MHz}, \mathrm{DMSO}-d_{6}\right): \delta 33.8\left(\mathrm{CH}_{2}\right), 55.5$, $57.6(\mathrm{OMe}), 89.8(\mathrm{C} 5), 123.2(\mathrm{C} 2), 127.8,130.9$ (aryl CH), 101.6, 110.2, 116.2, 130.1, 135.5, 137.9, 153.9, 154.4 (aryl C), $160.2(\mathrm{C}=\mathrm{O})$. HRMS (+ESI): $\mathrm{C}_{36} \mathrm{H}_{32} \mathrm{Cl}_{2} \mathrm{~N}_{4} \mathrm{O}_{6}[\mathrm{M}]^{+}$requires 686.1699, found 686.1693. (Found: $\mathrm{C}, 63.25 ; \mathrm{H}, 4.95 ; \mathrm{N}, 7.85 ; \mathrm{C}_{36} \mathrm{H}_{32} \mathrm{Cl}_{2} \mathrm{~N}_{4} \mathrm{O}_{6} 0.1 \mathrm{C}_{6} \mathrm{H}_{14}$ requires $\mathrm{C}, 63.14 ; \mathrm{H}, 4.84 ; \mathrm{N}, 8.05 \%)$.

N1,N3-Bis $\{[3-(4-c h l o r o p h e n y l)-4,6-d i m e t h o x y-1 H$-indol-7-yl]methyl\}adipic diamide (10). Adipoyl chloride $(0.06 \mathrm{~mL}, 0.41 \mathrm{mmol})$ in dry dichloromethane $(5 \mathrm{~mL})$ was added dropwise to a solution of 7-aminomethylindole $6(0.21 \mathrm{~g}, 0.65 \mathrm{mmol})$ in dry dichloromethane $(10 \mathrm{~mL})$ containing triethylamine $(0.09 \mathrm{~mL}, 0.65 \mathrm{mmol})$ and the reaction mixture stirred at room temperature for $1 \mathrm{~h}$. The solvent was then evaporated and the residue was quenched with water. The resulting precipitate was filtered, dried and recrystallised from methanol to yield the title compound $10(0.19 \mathrm{~g}, 86 \%)$ as a white solid. Mp 258-260 ${ }^{\circ} \mathrm{C}$. IR $(\mathrm{KBr}): v_{\max } 3314,2936,2839$, 1612, 1536, 1428, 1326, 1218, 1157, $788 \mathrm{~cm}^{-1}$. UV-vis (THF): $\lambda_{\max } 229 \mathrm{~nm}\left(\varepsilon 83,300 \mathrm{~cm}^{-1} \mathrm{M}^{-1}\right)$, 284 (37,770). ${ }^{1} \mathrm{H}$ NMR (300 MHz, DMSO- $\left.d_{6}\right): \delta 1.47\left(\mathrm{~s}, 4 \mathrm{H}, \mathrm{CH}_{2}\right), 2.10\left(\mathrm{~s}, 4 \mathrm{H}, \mathrm{CH}_{2}\right), 3.81(\mathrm{~s}$, $6 \mathrm{H}, \mathrm{OMe}), 3.86(\mathrm{~s}, 6 \mathrm{H}, \mathrm{OMe}), 4.44$ (d, J $\left.5.4 \mathrm{~Hz}, 4 \mathrm{H}, \mathrm{CH}_{2}-\mathrm{NH}\right), 6.43$ (s, 2H, H5), 7.28 (d, J 2.4 $\mathrm{Hz}, 2 \mathrm{H}, \mathrm{H} 2), 7.29-7.57\left(\mathrm{~m}, 8 \mathrm{H}\right.$, aryl H), $8.27(\mathrm{~m}, 2 \mathrm{H}, \mathrm{NH}), 10.91(\mathrm{~d}, J 2.1 \mathrm{~Hz}, 2 \mathrm{H}, \mathrm{NH}) .{ }^{13} \mathrm{C}$ NMR (75 MHz, DMSO- $\left.d_{6}\right): \delta 25.4,35.2\left(\mathrm{CH}_{2}\right), 32.9\left(\mathrm{CH}_{2}-\mathrm{NH}\right), 55.6,57.4(\mathrm{OMe}), 89.6(\mathrm{C} 5)$, $123.2(\mathrm{C} 2), 127.8,130.9$ (aryl CH), 102.8, 110.0, 116.2, 130.9, 135.5, 138.3, 153.8, 154.1 (aryl C), $173.7(\mathrm{C}=\mathrm{O})$. HRMS (+ESI): $\mathrm{C}_{40} \mathrm{H}_{40} \mathrm{Cl}_{2} \mathrm{~N}_{4} \mathrm{O}_{6}[\mathrm{M}]^{+}$requires 743.2403, found 743.2403. (Found: $\mathrm{C}, 64.03 ; \mathrm{H}, 5.44 ; \mathrm{N}, 7.39 ; \mathrm{C}_{40} \mathrm{H}_{40} \mathrm{Cl}_{2} \mathrm{~N}_{4} \mathrm{O}_{6} 0.05 \mathrm{CH}_{2} \mathrm{Cl}_{2}$ requires $\mathrm{C}, 64.32 ; \mathrm{H}, 5.40 ; \mathrm{N}$, $7.49 \%)$.

1-(3-(4-Chlorophenyl)-4,6-dimethoxy-1H-indol-7-yl)-N-((3-(4-chlorophenyl)-4,6-dimethoxy$1 \mathrm{H}$-indol-7-yl)-methylene)-methanamine (12). A mixture of 7-aminomethylindole $6(0.14 \mathrm{~g}$, $0.45 \mathrm{mmol})$ and 7-carbaldehyde $11(0.14 \mathrm{~g}, 0.45 \mathrm{mmol})$ was heated under reflux in absolute ethanol $(30 \mathrm{~mL})$ for $24 \mathrm{~h}$. The precipitate obtained was filtered, washed with water and dried. The crude product was purified by flash chromatography using dichloromethane/ethyl acetate (90:10) as eluent to yield the title compound $12(0.21 \mathrm{~g}, 75 \%)$ as a white solid. Mp 221-223 ${ }^{\circ} \mathrm{C}$. IR (KBr): $v_{\max } 3453,1623,1594,1341,1216,1146,1093,788 \mathrm{~cm}^{-1}$. UV-vis (THF): $\lambda_{\max } 225 \mathrm{~nm}$ ( $\left.\varepsilon 85,430 \mathrm{~cm}^{-1} \mathrm{M}^{-1}\right), 307(35,430) .{ }^{1} \mathrm{H}$ NMR $\left(300 \mathrm{MHz}, \mathrm{CDCl}_{3}\right): \delta 3.82$ (s, $\left.3 \mathrm{H}, \mathrm{OMe}\right), 3.89(\mathrm{~s}, 3 \mathrm{H}$, $\mathrm{OMe}$ ), 3.90 (s, 3H, OMe), 3.94 (s, 3H, OMe), 5.02 (s, 1H, $\mathrm{CH}_{2}$ ), 6.46 (s, 1H, H5), 6.49 (s, 1H, H5'), 7.30-7.59 (m, 10H H2+H2', aryl H), 8.91 (s, 1H, CH), 11.38 (d, J 2.3 Hz, 1H, NH), 11.49 (bs, $1 \mathrm{H}, \mathrm{NH}$ ). HRMS (+ESI): $\mathrm{C}_{34} \mathrm{H}_{29} \mathrm{Cl}_{2} \mathrm{~N}_{3} \mathrm{O}_{4}[\mathrm{M}+\mathrm{H}]^{+}$requires 614.1569, found 614.1599. The sample was not soluble enough for ${ }^{13} \mathrm{C}$ NMR measurement. 
Bis-((3-(4-chlorophenyl)-4,6-dimethoxy-1H-indol-7-yl)methyl)amine (13). To a solution of bis-indole $12(0.08 \mathrm{~g}, 0.13 \mathrm{mmol})$ in a mixture of absolute ethanol/tetrahydrofuran $(30 \mathrm{~mL})(1: 1)$, sodium borohydride $(1.00 \mathrm{~g}, 26.3 \mathrm{mmol})$ was added and the mixture was heated under reflux for $8 \mathrm{~h}$. The solvent was removed under reduced pressure, and the residue was treated with water and neutralized using dilute hydrochloric acid $(2 \mathrm{M})$. The resulting precipitate was filtered, dried and recrystallised from dichloromethane/n-hexane to afford the title compound $13(0.05 \mathrm{~g}, 65 \%)$ as a white solid. Mp 134-136 ${ }^{\circ} \mathrm{C}$. IR (KBr): $v_{\max } 3307,2933,2836,1622,1597,1540,1518$, 1463, 1329, 1201, $1123 \mathrm{~cm}^{-1}$. UV-vis (THF): $\lambda_{\max } 305 \mathrm{~nm}\left(\varepsilon 29,800 \mathrm{~cm}^{-1} \mathrm{M}^{-1}\right) .{ }^{1} \mathrm{H}$ NMR $(300$ $\mathrm{MHz}_{\mathrm{CDCl}}$ ): $\delta 3.74$ (s, 6H, OMe), $3.78(\mathrm{~s}, 6 \mathrm{H}, \mathrm{OMe}), 4.01\left(\mathrm{~s}, 4 \mathrm{H}, \mathrm{CH}_{2}\right) 6.23(\mathrm{~s}, 2 \mathrm{H}, \mathrm{H} 5), 6.83$ $(\mathrm{s}, 2 \mathrm{H}, \mathrm{H} 2), 7.22,7.41\left(2 \mathrm{~d}, J 8.6 \mathrm{~Hz}, 8 \mathrm{H}\right.$, aryl H), $9.29(\mathrm{bs}, 2 \mathrm{H}, \mathrm{NH}) .{ }^{13} \mathrm{C} \mathrm{NMR}(75 \mathrm{MHz}$, $\left.\mathrm{CDCl}_{3}\right): \delta 42.9\left(\mathrm{CH}_{2}\right), 55.3,57.0(\mathrm{OMe}), 88.9(\mathrm{C} 5), 121.7(\mathrm{C} 2), 127.7,130.6(\operatorname{aryl} \mathrm{CH}), 110.6$, 117.4, 131.4, 134.6, 138.5, 154.1, 154.5 (aryl C). HRMS (+ESI): $\mathrm{C}_{34} \mathrm{H}_{31} \mathrm{Cl}_{2} \mathrm{~N}_{3} \mathrm{O}_{4}[\mathrm{M}+\mathrm{H}]^{+}$ requires 616.1770 , found 616.1768 .

\section{Acknowledgements}

We thank the University of New South Wales and the Turkish Government for their financial support.

\section{References}

1. Moron, J. A.; Campillo, M.; Perez, V.; Unzeta, M.; Pardo, L. J. Med. Chem. 2000, 43, 16841691.

2. http://dx.doi.org/10.1021/jm991164x

3. Csomos, P.; Fodor, L.; Sohar, P.; Bernath, G. Tetrahedron 2005, 61, 9257-9262. http://dx.doi.org/10.1016/j.tet.2005.07.068

4. Pedras, M. S. C.; Jha, M.; Ahiahonu, P. W. K. Curr. Org. Chem. 2003, 7, 1635-1647. http://dx.doi.org/10.2174/1385272033486242

5. Hollingsworth, M. D.; Ward, M. D. Chem. Mater. 1994, 6, 1087-1093. http://dx.doi.org/10.1021/cm00044a001

6. Somei, M.; Yamada, F.; Morikawa, H. Heterocycles 1997, 46, 91-94. http://dx.doi.org/10.3987/COM-97-S33

7. Wahyuningsih, T. D.; Kumar, N.; Nugent, S. J.; Black, D. StC. Tetrahedron 2005, 61, 10501-10506.

8. http://dx.doi.org/10.1016/j.tet.2005.08.049

9. Humphrey, J. M.; Chamberlin, A. R. Chem. Rev. 1997, 97, 2243-2266. http://dx.doi.org/10.1021/cr950005s 
10. Jones, A. W.; Purwono, B.; Bowyer, P. K.; Mitchell, P. S. R.; Kumar, N.; Nugent, S. J.; Jolliffe, K. A.; Black, D. StC. Tetrahedron 2004, 60, 10779-10786. http://dx.doi.org/10.1016/j.tet.2004.08.086

11. Bowyer, P. K.; Black, D. StC.; Craig, D. C.; Rae, A. D.; Willis, A. C. J. Chem. Soc., Dalton Trans. 2001, 1948-1958. http://dx.doi.org/10.1039/b101350p

12. Sommers, A. H.; Aaland, S. E. J. Org. Chem. 1956, 21, 484-485. http://dx.doi.org/10.1021/jo01110a612 